\title{
Improving Image Quality Using Filtering Weighted Median Filter Techniques on Magnetic Resonance Imaging (MRI) Breast Images
}

\author{
$* 1^{\text {st }}$ Aulia Annisa \\ Postgraduate Program in Medical \\ Imaging Diagnostic \\ Health Polytechnic Semarang \\ Semarang, Indonesia \\ aulia14annisa@gmail.com
}

\author{
$2^{\text {nd }}$ Leny Latifah \\ Magelang Health Research and \\ Development Center \\ Ministry of Health Republic of \\ Indonesia \\ Magelang, Indonesia \\ lenylatifah1@gmail.com
}

\author{
$3^{\text {rd }}$ Luthfi Rusyadi \\ Postgraduate Program in Medical \\ Imaging Diagnostic \\ Health Polytechnic Semarang \\ Semarang, Indonesia \\ luthfirusyadi@yahoo.com
}

\begin{abstract}
Acquisition of MRI images takes a long time so that it can cause movements that create motion images in the image. In addition to the acquisition of MRI itself can produce noise, movement of the image can reduce the quality of the image. The image quality can be improved by using denoising post processing methods. One denoising method that can improve image quality is Weighted Median Filter (WMF). Data was taken from MRI Breast patient images and then given the application of WMF filtering technique. Denoising post-image acquisition becomes a cheaper and effective alternative. This research is expected to be able to improve the image quality on MRI Breast axial T2WI sequences with noise reduction.
\end{abstract}

Keywords- MRI Breast, Weighted Median Filter (WMF), SNR, CNR

\section{INTRODUCTION}

Breast imaging is one of the key techniques in the successful detection, diagnosis, and treatment of breast cancer[1]. Breast tumors are divided into two, benign and malignant tumors. Benign tumors can be mammary fibroadenomas, breast cysts, fibrocystic breasts, fat necrosis and intraductal papillomas while malignant tumors are breast cancer [2] [3]. Breast cancer is one of the leading causes of cancer deaths in women throughout the world. The incidence of cancer in Indonesia in 2018 is around 136.2 per 100,000 population, which is ranked 8th in Southeast Asia [4].

One of the tests to detect breast tumors is MRI Breast. Magnetic resonance imaging (MRI) of the breast is being performed more frequently to improve primary and recurrent tumor detection, characterization, and patient's response to therapy. Sensitivity approaches 90\%.[5] Specificity ranges from $37 \%$ to $100 \%$.[1] MRI Breast is done further to evaluate abnormalities that are difficult to assess and see on mammography [5] [6] [7] Breast MRI is not a substitute for mammography and ultrasonographic imaging, but is an additional tool that has valuable information about many breast conditions[5]

MRI Breast Imaging maximizes sensitivity and specificity for breast cancer while minimizing scan time. Longer overall scan time must be avoided, because it can cause discomfort and movement of the patient, which causes artifacts and secondary image degradation due to motion [8].

Images obtained by acquisition devices are vulnerable to the environment. Image recovery tries to minimize the effects of this degradation through filters [9][10]. Therefore, a fundamental problem in image processing is improving its quality through noise reduction [52]. A variety of techniques dedicated to carrying out this task exist. Each depends on the type of noise in the image. Noise not only degrades image quality but can also cause feature extraction, analysis and recognition algorithms to become unreliable. MRI images are usually affected by a type of noise called gaussian noise. The presence of noise inhibits diagnosis. Diagnostic and visual quality of MR images is affected by noise added at acquisition[9].

MRI Breast provides good breast anatomy information including the location of 3D lesions, but has limitations to characterize tissue [12]. Contrast dynamic magnetic resonance imaging appears as an important technique that provides pharmacokinetic parameters. In this technique the acquisition time is longer so as to result in patient discomfort and a longer analysis time for the doctor which affects the overall assessment[12]

The T2WI sequence was chosen because, in the MRI examination Breast included non-fat T1WI sequence, fatsuppressed T2WI scan and dynamic contrast scanning. Recent studies show the value of Diffusion Weighted Images (DWI) and Magnetic Resonance Spectroscopy (MRS) on MRI Breast provide additional information that can help in the diagnosis and differentiation of breast lesions, but the two sequences are not routinely used in clinical breast MRI due to limitations and challenges technical[13].

Impairment of image quality will reduce the doctor's reading perception or diagnosis and the occurrence of noise in the image [14] Noise will cause the pixel intensity value does not reflect the actual pixel intensity value [15]. Denoising methods that can be applied to improve image quality, including the Weighted Median Filter (WMF) [16] [17 [18]. 
In research on the detection of breast tumors in mammography, it is said to be necessary to do research related to the improvement of image quality in breast tumor cases [19]. Research using wavelet-based WMF filters has been carried out on MRI Brain examination and the results are that wavelet-based median filters tend to produce good denoisa images in noise reduction [9].

\section{RELATED WORKS}

Noise reduction (denoising) on MRI images can be done by two methods, namely during image acquisition and after image acquisition [20]. Denoising has an important role in the medical field before the image is analyzed. Denoising aims to eliminate noise by maintaining important information and the original resolution of the image without causing artifacts [20] [21] [22]. The application of denoising to images will increase the Signal-to-Noise Ratio (SNR) and improve image quality[21].

\section{A. Weighted Median Filter (WMF}

Weighted Median Filter is a derivative of a median filter. The previously designed weighted median filter gives more weight to some values in the window while the center weighted median filter gives more weight to the window center value making it easier to design and implement than other weighted median filters [18].

Weighted median filters (WM) were first introduced as generalizations of standard median filters, where nonnegative integer weights were assigned to each position in the filter window [23] [24]. For real value signals, WM filters can be defined in two different but equal ways. The first definition can be used in the general case of positive integer weights [25]. The median filter is mostly used by researchers because of its ability to provide excellent noise reduction with less blur for various types of noise [27].

\section{B. Signal To Noise Ratio (SNR)}

What is meant by SNR is the ratio between the magnitude of the signal amplitude with the amplitude of noise [8] [26] This SNR is also strongly related to the amount of X-ray energy used per pixel in the image. If one attempts to reduce the pixel size (causing an increase in Spatial Resolution) and the dose level is kept the same, the number of X-rays per pixel is reduced.37 To calculate the SNR the Region of Interest (ROI) value is determined using the following equation:

$$
S N R=\frac{P V_{V}}{S D_{B}}
$$

Where $P V_{v}$ is the average HU value of the object, the standard deviation of the HU value of the background.

\section{Contrast To Noise Ratio(CNR)}

CNR is the SNR difference between organs that are close together. A good CNR can show differences in pathological areas in healthy areas. Contrast is a major component in the image that carries information. The choice of imaging modality and imaging technique is basically the determination of contrast to obtain information.
A more suitable assessment of the effect of noise on image information is CNR [36] which can be written:

$$
C N R=\frac{H u \text { Obyek }-H u \text { Background }}{\text { o'Background }}
$$

In this equation the contrast is only the difference in intensity between the object and its background object divided by the standard deviation of the background [4].

\section{ProposedMethodology}

This type of research is analytical research with a numerical measurement scale in one pair group. It is said to be in pairs because the data is measured twice in the same individual. So that is determined by the formula Machin et al (2009) and obtained a sample of 15.

This research was conducted on MRI examination of T2WI sequences. In the MRI Breast T2WI SPAIR Image, image quality improvement was performed with a Denoising Weighted Median Filter (WMF) technique to produce better quality images. Each image is measured before and after the image denoising technique is measured. Image quality is assessed in two ways. namely measurement objectively by measuring the image by measuring SNR and CNR.

The study began with MRI breast scanning on axial T2WI SPAIR sequences. Cittra MRI Breast T2WI SPAIR then performed denoising Weighted Median Filter (WMF) techniques. This step aims to determine the changes before (pre-test) and after (post-test) treatment of denoising WMF. The type of pre-experimental research uses the One group pretest and post-test without control design.

\section{A. WMF Filtering Techniques}

In this study the application of the denoising Weighted Median Filter (WMF) technique was carried out at the postprocessing stage of the image. The initial stage is looking for image data from the results of examinations in MRI Breast patients with axial T2WI SPAIR sequences. The next step is to store images in the form of Digital Imaging and Communication in Medicine (DICOM) on CD / DVD. The image is stored in the form of Digital Imaging and Communication in Medicine(DICOM).

Image MRI Breast T2WI SPAIR sequence that has been stored on DVD_R, then the image quality is improved. Noise in the image is made to reduce noise and increase contrast with denoising Weighted Median Filter (WMF) techniques. The image is stored in the form of Digital Imaging and Communication in Medicine (DICOM. Noise in the image is done to reduce noise and increase contrast with the denoising technique. Weighted Median Filter (WMF).

Identification of MRI Breast imagery with the MATLAB program, is done by providing denoising techniques with the application of Weighted Median Filter (WMF) with convolution techniques, namely the multiplication of two matrices, image matrix elements and filter matrix elements with the operation of shifting the pixel by pixel filter window, then stored in a matrix per pixel filter window. new matrix. After treatment with WMF, the image is analyzed and evaluated visually subjectively and objectively. Filtering operation is one of the basic tools provided by mathlab for image processing by reducing noise 
or certain fine grains in the image. WMF Filters sort pixel values and their constancy from the smallest to the largest then the middle value is obtained.

The steps of the filtering process are as follows:

- Input syintax filter weighted median filter on the GUI available in the mathlab application

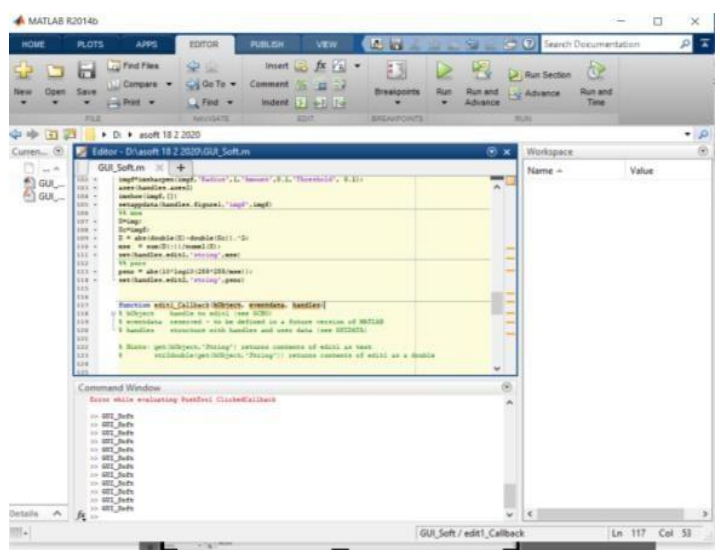

Fig. 1. Input syntax filter weighted median filter on theGU

- Doing running and after running out the GUI window display filtering process

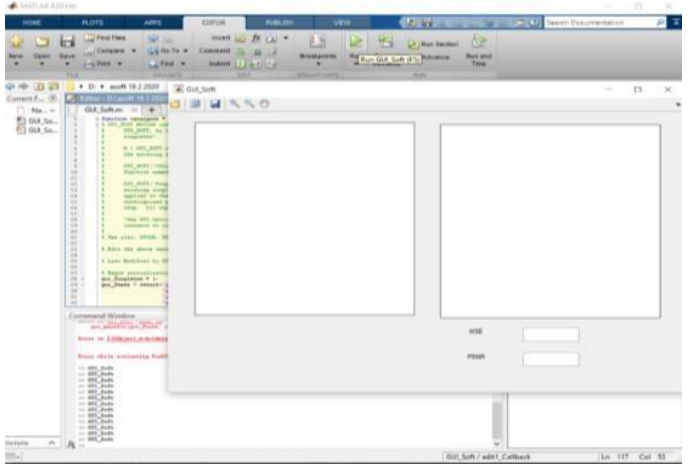

Fig. 2. GUI toolbox display after running

- After the GUI window exits the filtering process, input the original image to be filtered then click the filter button on the toolbar window and will display the results of the WMF Denoising process after filtering

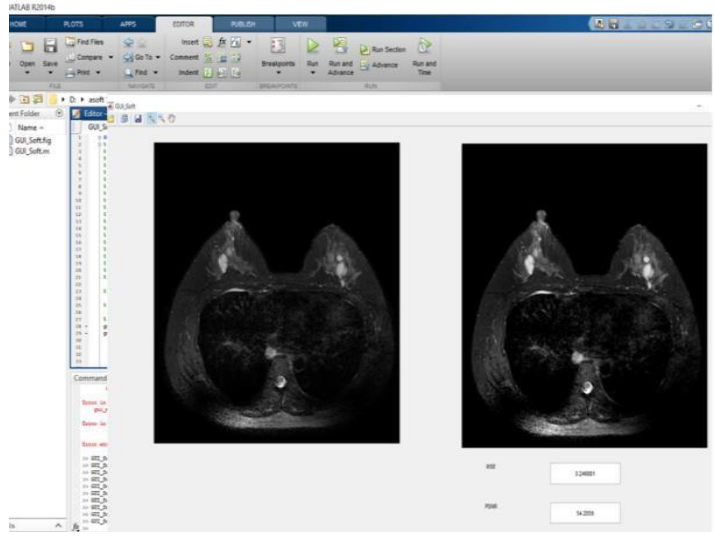

Fig. 3. Displays the results of the WMF Denoising process image after filtering
Digital image can be defined as a function of two variables $\mathrm{f}(\mathrm{x}, \mathrm{y})$ where $\mathrm{x}$ and $\mathrm{y}$ are spatial coordinates and the value $f(x, y)$ is the intensity of the image at these coordinates. Image processing is an image processing and analysis process that mostly involves visual perception. This process has the characteristics of input data and output information in the form of many images. The term digital image processing is generally defined as two-dimensional image processing with a computer. In a broader definition, digital image processing also includes all two-dimensional data. Digital images are rows of real and complex numbers represented by certain bits.

The format of digital image data is closely related to color. In most cases, especially for the purpose of visual appearance, digital data values represent the colors of the processed image. The most widely used digital image formats are Binary (Monochrome) imagery, Gray Scale Image (Grayscale), true color image, and Indexed Color Image. In the color image, each point has a specific color which is a combination of three colors: red, green, blue or often called RGB image, each base color has its own intensity with a maximum value of 255 . Filtering operation is one of the basic tools provided by mathlab for image processing by reducing noise or certain fine grains in the image.

\section{RESUlT AND DiSCUSSION}

A pre-experimental analytic study has been carried out with the application of the denoising Weighted Median Filter (WMF) technique on MRI Breast images of T2WI sequences Axialslices.

In this study the selected MRI Breast Image is saved to CD / DVD in Dicom format, all MRI parameters are controlled to remain the same in each sample. The MRI image of the Berast T2WI SPAIR sequence was then repaired image information using a denoising Weighted Median Filter (WMF) technique. This research was conducted to assess the quality of MRI Breast imagery by calculating the SNR and CNR values.

\section{A. Signal To Noise Ratio(SNR)}

SNR is the ratio between the magnitude of the signal amplitude and the noise amplitude. To calculate the SNR value, the Region of Interest (ROI) is determined as shown below:

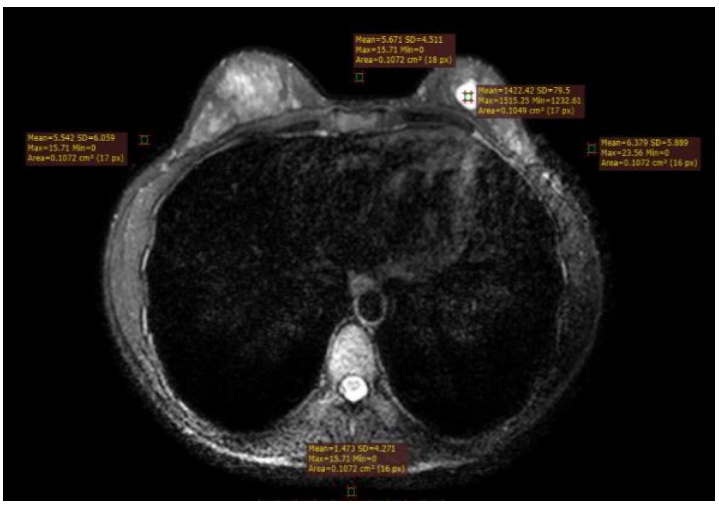

Fig. 4. ROI Points for SNR assessment on Image before WMF 


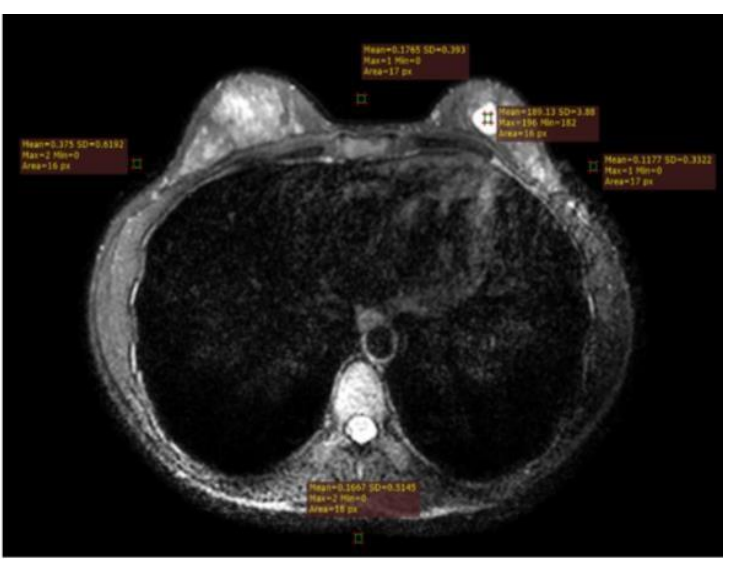

Fig. 5. ROI Points for SNR assessment on Image after WMF

The average Signal to Noise Ratio (SNR) value on MRI Breast T2WI SPAIR images between before and after the application of WMF denoising can be seen in the following table:

TABLE I. SNR VALUES OF MRI BREAST IMAGERY

\begin{tabular}{lcc}
\hline \multirow{2}{*}{ Image } & \multicolumn{2}{c}{ SNR } \\
\cline { 2 - 3 } & Before & After \\
\hline Image 1 & 129,22 & 616,76 \\
Image 2 & 237,93 & 1575,68 \\
Image 3 & 117,24 & 1697,25 \\
Image 4 & 40,11 & 677,6 \\
Image 5 & 57,73 & 1329,25 \\
Image 6 & 79,51 & 1237,22 \\
Image 7 & 75,76 & 1043,54 \\
Image 8 & 61,66 & 613,76 \\
Image 9 & 38,82 & 477,59 \\
Image 10 & 88,96 & 1087,86 \\
Image 11 & 45,66 & 722,32 \\
Image 12 & 107,17 & 344,23 \\
Image 13 & 85,23 & 342,0 \\
Image 14 & 26,096 & 1312,57 \\
Image 15 & 76,84 & 783,52 \\
\hline TOTAL & 84,529 & 924,076 \\
\hline
\end{tabular}

SNR values before denoising ranged from an average of 84.529 and SNR values after denoising ranged from an average of 924,076 . Data normality tests were performed using Shapiro Wilk before conducting SNR different tests before and after denoising WMF shown in the following table:

TABLE II. SNR NORMALITY TEST

\begin{tabular}{ccc}
\hline SNR Normality & $\begin{array}{c}\boldsymbol{P} \text { Value } \\
\text { shapiro wilk }\end{array}$ & Information \\
\hline SNR Pre & 0,009 & $\begin{array}{c}\text { Data not } \\
\text { normally } \\
\text { distributed }\end{array}$ \\
\hline
\end{tabular}

Based on the Wilcoxon different test results on the SNR value of the MRI Breast image of the T2WI SPAIR sequence before and after the application of WMF denoising techniques, the $\mathrm{p}$ value was 0.001 . The conclusion from the results of this study there is a significant difference between the image before it is reduced to the image after it has been reduced by WMF denoising techniques.
The image after being improved by eliminating noise and improving image quality by using $\mathrm{WMF}$ denoising. If the noise value decreases, the dividing factor of the SNR will be small so that the value of the SNR will increase58. Denoising Weihted Median Filter (WMF) uses the redundancy of information contained in the image to eliminatenoise.

The stored voxel intensity values are calculated as a weighted average of all voxel intensities in figure 7. SNR values obtained from MRI are usually used to make comparisons between hardware imaging, imaging protocols, and sequences. (Welvaert et al, 2013). Signals can be increased and lowered for relative noise. Increasing the signal can increase the SNR, conversely decreasing the signal can decrease theSNR.

\section{B. Contrast To Noise Ratio(CNR)}

CNR is the SNR difference between organs that are close together. A good CNR can show pathological differences in healthy areas[20]. The average value of Contrastl to Noise Ratio (CNR) on MRI Breast T2WI SPAIR images between before and after the application of WMF denoising can be seen in the following table:

TABLE III. CNR VALUES OF MRI BREAST IMAGES

\begin{tabular}{lcc}
\hline \multirow{2}{*}{ Image } & \multicolumn{2}{c}{ CNR } \\
\cline { 2 - 3 } & Before & After \\
\hline Image 1 & 222.45 & 433,76 \\
Image 2 & 337,39 & 969,19 \\
Image 3 & 249,27 & 1046,80 \\
Image 4 & 97,50 & 312,78 \\
Image 5 & 117,12 & 734,72 \\
Image 6 & 164,37 & 907,16 \\
Image 7 & 172,56 & 565,07 \\
Image 8 & 143,11 & 485,42 \\
Image 9 & 68,53 & 432,61 \\
Image 10 & 235,38 & 960,96 \\
Image 11 & 172,26 & 1135,96 \\
Image 12 & 158,21 & 236,26 \\
Image 13 & 188,55 & 365,12 \\
Image 14 & 199,77 & 1827,68 \\
Image 15 & 111,95 & 470,4 \\
\hline TOTAL & 172,56 & 725,59 \\
\hline
\end{tabular}

The CNR value before the application of denoising WMF ranged on average to 172.56 , while the $\mathrm{CNR}$ value after the application of denoising WMF ranged at an average of 725.59. Data normality test was performed using Shapiro Wilk before conducting different CNR tests before and after denoising WMF shown in the following table:

TABLE IV. NORMALITY TEST FOR CNR DATA

\begin{tabular}{llll}
\hline $\begin{array}{c}\text { Normalitas Data } \\
\text { CNR }\end{array}$ & \multicolumn{2}{c}{$\begin{array}{c}\text { P Value shapiro } \\
\text { wilk }\end{array}$} & \multicolumn{2}{c}{ Information } \\
\hline CNR Pre & 0,792 & $\begin{array}{l}\text { Data } \\
\text { normally } \\
\text { distributed }\end{array}$ & not \\
CNT -Post & 0,349 & \multicolumn{2}{c}{} \\
\hline
\end{tabular}


TABLE V. PAIRED T-TEST OF CNR

\begin{tabular}{ccclcr}
\hline CNR & Mean & $\begin{array}{c}\text { P value } \\
\text { Paired T test }\end{array}$ & Significance \\
\hline CNR pre & 172,56 & 0,000 & $\begin{array}{l}\text { There is a } \\
\text { difference }\end{array}$ & \\
CNR post & 725,59 & & &
\end{tabular}

From the results of different tests showed differences in CNR values on MRI Breast T2WI SPAIR images between before and after the application of WMF denoising technique with $p$ value 0,000 . This value is smaller than

$0.05(\mathrm{p}=0.000<0.05)$, so Ho is accepted. Conclusion there are significant differences between CNR values before and after the application of WMF denoising techniques.

The increase in CNR value in the image after the application of WMF denoising technique is caused due to the decrease in the noise value in the image after the reduction, so in this study, the conclusion is that there are differences before and after the reduction of the WMF denoising technique. Noise on MRI can arise as a result of the acquisition process of image acquisition and acquisition using hardware, transmission errors such as the acquisition of array coils, gradient coils and fields in homogeneity and patient-based, such as physiological noise including body movements, heart rate or respiratory movements. Noise depends on several factors Signal, scan time, spin magnetization, slice thickness, object size and algorithm, for example MRI images obtained using coils with a parallel channel system (multiple channel coils) cause very inhomogeneous noise, where the signal obtained in K-space becomes damaged in the presence of complex additive Caussian noise. This noise will affect the resolution contrast, if the noise is lower the resolution contrast will increase.

Low noise causes high spatial resolution. Spatial resolution that will increase between 2 different objects will be clear and firm. A good CNR can show differences in pathological areas in healthy areas. Filtering in particular the median filter used is WMF can reduce noise but cannot eliminate it.

\section{CONCLUSION}

Good image quality will support visual assessment of anatomic information better. SNR assessment of images that have been obtained can be continued to strengthen the assessment of breast image anatomy information. The mean rank results of the different SNR assessment tests show that the image has increased SNR after denoising WMF. The use of WMF denoising technique application method on MRI Breast image of T2WI sequences axial pieces to reduce noise based on computer programming is able to produce better quality MRI Breast image of T2WI sequences, so that the use of WMF denoising technique using matlab program can be used as an alternative post processing tool for MRI examination Breast sequence T2WI axial pieces.

WMF denoising techniques can reduce noise, improve image quality and image information should be used in sequences that cause a lot of noise such as Gradient Echo (GRE) and in other MRI examinations especially in examinations that require low scan time.

Utilization of computer programming matlab can be used not only in noise reduction, but can be used to assess or diagnose an abnormality of other organs in the medical. Especially in this study for breast cancer cases segmentation can be done for the detection and identification of tumors.

\section{REFERENCES}

[1] Lee $\mathrm{CH}$. Problem solving MR imaging of the breast. Radiol Clin North Am2004;42:919-34

[2] Soetrisno E,Nasar IM, Himawan S, Marwoto W. 2010. Payudara. Dalam: Buku ajar patologi II. Edisi ke-1. Jakarta: Sagung Seto.hlm156-78

[3] Rosai, Juan., 2002. Anatomi Payudara. Dalam: Ackerman"s Surgical Pathology.Valume 1. 9thedition.Mosby.

[4] Kementerian Kesehatan Republik Indonesia. 2019. Data Hari Kanker Sedunia.www.depkes.go.id

[5] Radiological Society of North America, reviewed by an ACR (American College of Radiology) - RSNA (Radiological Society of North America) committee: "Magnetic Resonance Imaging-Breast", comprising physicians with expertise in several radiologic areas. Reviewed: Feb-15-2018. 2019 Radiological Society of North America,Inc

[6] Eren D.eh,MD, dkk. 2014. Positioning in breast MR Imaging to Optimize Image Quality. accepted May 29 For this journal-basedSA-

[7] CME. www.rsna.org/rsnarights

[8] Shandong Wu, Susan P. Weinstein, Emily F. Conant, Mitchell D. Schnall, and Despina Kontos. 2013. Automated chest wall line detection for whole-breast segmentation in sagittal breast MR images. Medical Physics, Vol. 40, No.4

[9] Westbrook C, Carolyne ,K Roth dan Talbot, J, 2011, MRI inPractice,

[10] Fourth Edition. Blackwell Science. Ltd., United Kingdom

[11] N. Rajalakshmi, K. Narayanan, P. Amudhavalli. 2018. WaveletBased Weighted Median Filter for Image Denoising of MRI Brain Images : Indonesian Journal of Electrical Engineering and Computer Science

[12] D.L. Donoho., Denoise by softthresholding, “ IEEE Transactions on Information Theory, 41(1995), pp.613-627.

[13] R. Gonzalez and R. Woods, "Digital Image Processing using MATLAB," Second Edition, THM(2009).

[14] [N. Rajalakshmi, K. Narayanan, P. Amudhavalli. 2018. WaveletBased Weighted Median Filter for Image Denoising of MRI Brain Images : Indonesian Journal of Electrical Engineering and Computer Science

[15] Amarnath Jena DNB (NM)Shashi Bhushan Mehta PhDSangeeta Taneja MD.Optimizing MRI scan time in the computation of pharmacokinetic parameters (Ktrans) in breast cancerdiagnosis.

[16] Chen Lin Clark, David Rogers, Shadie Majidi. 2015. Fat suppression techniques in breast magnetic resonance imaging: a critical comparison and state of the art. Department of Radiology and Imaging Science, Indiana University School of Medicine, Indianapolis, IN,USA

[17] Dale, M. Brian, M.A Brown dan Richard C. Semelka, 2015, MRI Basic Principle and Applications, Fifth Edition, John Wiley and Sons Inc : NewJersey

[18] Yang J, Fan J, Ai D, Zhou S, Tang S, Wang Y. Brain MR image denoising for Rician noise using pre-smooth non-local means filter. 2015

[19] More S, Hanchate V V. A Survey on Magnetic Resonance Image Denoising Methods. international Research Journal ofEngineering

[20] and Technology (IRJET)e-ISSN: 23950056Volume: 03 Issue: 05 | May-2016

[21] Manjón J V., Coupé P, Martí-Bonmatí L, Collins DL, Robles M. Adaptive non-local means denoising of MR images with spatially varying noise levels. J Magn Reson Imaging.2010;31(1):192-203

[22] Sandeep Kaur, Navdeep Singh. 2014. Image Denoising Techniques: A Review Risearch Penelitian, Magister Teknologi, Departemen Teknik Komputer, Universitas Punjabi,Patiala.

[23] R.Ramani, DR.N.Suthantira, S.Valarmathy. (2013). The PreProcessing Techniques for Breast Cancer Detection in Mammography Images. I.J.Image Graphics and SignalProcessing.

[24] DOI:10.5815/ijigsp.2013.05.06 
[25] Mohan J, Krishnaveni V, Guo Y. A survey on the magnetic resonance image denoising methods. Biomed Signal Process Kontrol. 2014;9(1):56-69.

[26] Vaishali S, Rao KK, Rao GVS. A review on noise reduction methods for brain MRI images. Int Conf Signal Process Commun Eng Syst Proc SPACES 2015, Assoc with IEEE.2015;(January):363-5.

[27] Anjanappa C, Sheshadri HS. Denoising of Rician noise in Magnitude MRI Images using wavelet shrinkage and fusion method. OSR Journal of VLSI and Signal Processing (IOSR-JVSP) Volume 6, Issue 5, Ver. II (Sep. -Oct.2016)
[28] D. R. K. Brownrigg, "The weighted median filter," Commun.ACM, vol. 27, no. 8, pp. 807-818, Aug. 1984.

[29] B. I. Justusson, "Median filtering: statistical properties," in TwoDimensional Digital Signal Processing /I, T. S. Huang, Ed. New York Springer-Verlag, 1981.

[30] Lin Yin, Ruikang Yang.1996.Weighted Median Filters: A Tutoria. Ieee Transactions On Circuits And Systems-11: Analog And Digital Signal Processing, Vol. 43, No.3

[31] Hashemi, H. Ray and Bradley, G. William, 2010, MRI :The Basic third edition, Williams \& Wilkins, USA. 